\title{
Calcium Homeostasis in Multiple Sclerosis
}

\section{두(ㅇ)(요}

Authors

Petra Hundehege, Lisa Epping, S. G. Meuth

\author{
Affiliation \\ Institute of Translational Neurology, Department of \\ Neurology, University Clinic Münster \\ Key words \\ pathology, immune cells, blood-brain barrier, central \\ nervous system, store-operated calcium entry \\ Bibliography \\ DOI https://doi.org/10.1055/s-0043-109031 \\ Neurology International Open 2017; 1: E127-E135 \\ (c) Georg Thieme Verlag KG Stuttgart · New York \\ ISSN 2511-1795 \\ Correspondence \\ Prof. Dr. med. Dr. rer. nat. Sven G. Meuth \\ Department of Neurology \\ Albert-Schweitzer-Campus 1
}

\author{
Building A1 \\ 48149 Münster \\ Germany \\ sven.meuth@ukmuenster.de
}

\section{Introduction}

Multiple sclerosis (MS) is a chronic-inflammatory demyelinating disease of the central nervous system (CNS). Essential pathogenic steps include the activation of myelin-autoreactive T-cells in the peripheral immune organs (e.g. spleen, lymph nodes), their penetration into the CNS, their local (re-)activation and proliferation, and the subsequent demyelination of neuronal axons [1]. In addition to damage caused to the myelin sheath and myelin-producing oligodendrocytes, neurons are destroyed in early stages of the disease [2]. Despite considerable efforts in recent decades, the causes of MS pathology are still not clear. Different studies have investigated the contribution of diverse immune or neuronal cell types $[3,4]$ as well as the influence of genetic factors (e. g. altered function of ion channels [5]) or environmental factors (e. g. UV irradiation [6]). In addition, there are indications for disturbances in calcium homeostasis in MS patients, contributing to MS pathogenesis. In general, calcium plays a central role in almost every process of the human body. Within cells, calcium acts as a secondary messenger in signal transduction pathways. Under physiological conditions, the concentration of free and thus active calcium is highly regulated and kept low both extra- and intracellularly (intracellu- larly approx. $100 \mathrm{nM}$ free calcium). To maintain low concentrations, calcium ions are actively pumped from the cytosol into the extracellular space, the endoplasmic reticulum (ER) and sometimes into the mitochondria. Certain proteins of the cytoplasm and cell organelles additionally act as buffers by binding free calcium [7]. The intracellular calcium level is only briefly increased when required (e. g., after activation of immune cells or release of neurotransmitters in the CNS). Calcium ions flow into the cytosol via two main pathways: 1) along their electrochemical gradient from the extracellular space through calcium channels in the plasma membrane and 2) from intracellular calcium stores such as the ER, which constantly accumulate calcium ions and release them during certain cellular events (the latter is better known as store-operated calcium entry (SOCE) [7]).

Balanced calcium homeostasis is essential for the efficient function of cells and organisms. This overview article summarizes the current knowledge of calcium-dependent mechanisms in MS pathology. In particular, we specifically discuss disturbed calcium signals in various compartments of MS pathogenesis: the peripheral immune system, the blood-brain barrier (BBB) and neurons in the CNS. 
CALCIUM: ONE OF THE MOST IMPORTANT BULK ELEMENTS IN THE HUMAN BODY

Making up $1-1.1 \mathrm{~kg}$ of body weight, calcium is the most abundant mineral in the human organism. The teeth and bones contain $99 \%$ of the calcium in the body. Outside the cells, calcium is involved in blood coagulation and maintenance of cell membranes. Intracellularly, calcium $\left(\mathrm{Ca}^{2+}\right)$ acts as a messenger in many signalling pathways. Thus, calcium is involved in the excitation of muscles and nerves, glycogen metabolism, cell division, as well as activation of some enzymes and hormones. The influx of calcium ions into muscle cells leads to muscle contraction. A concentration of $2.1-2.6 \mathrm{mmol} / \mathrm{l}$ of calcium must always be present in the blood. Calcium ions are released from the bone into the bloodstream under controlled conditions and transported as dissolved ions or bound to proteins such as serum albumin. Parathormone secreted by the parathyroid gland promotes calcium release from the bone. Calcitriol, the active form of vitamin D3, promotes the absorption of calcium from the intestine and the mobilization of calcium ions from the bone matrix. Calcitonin, which is secreted by the parafollicular cells of the thyroid, also affects the calcium level. Only about $50 \%$ of extracellular calcium is present as free-ionized calcium and thus in its biologically active form.

\section{APPROVED MS DRUGS AND THEIR EFFECT ON CALCIUM HOMEOSTASIS}

In everyday clinical practice, MS medication for symptomatic therapy is used, which directly or indirectly affects the calcium homeostasis of different cell types. The centrally effective muscle relaxant baclofen is a derivative of $y$-aminobutyric acid (GABA) and binds as a specific agonist to GABA $B$ receptors. This binding induces inhibitory postsynaptic potentials and thus suppresses neuronal activity while indirectly increasing intracellular calcium levels. In addition to this indirect mechanism, baclofen also has a direct effect, since it binds a special subunit of the voltage-dependent calcium channels and thus blocks the influx of calcium. This effective mechanism has also been described for pregabalin, which is prescribed to MS patients for the relief of neuropathic pain. Thus, pregabalin, like baclofen, is a GABA analogue but paradoxically - and unlike baclofen - has no affinity for GABA receptors. However, like baclofen, it binds directly to specific, voltage-dependent calcium channels, which reduces the release of neurotransmitters into the synaptic cleft. In MS therapy, 4-aminopyridine (4-AP) is used to improve ambulatory ability. In general, 4-AP inhibits potassium channels and thereby inducing the stimulation of calcium channels. Subsequently, it enhances the duration of action potentials, calcium influx into neurons, and neurotransmitter release. Recently, however, it has also been shown that 4-AP has a direct influence on voltage-dependent P/Q- and N-type calcium channels. Dantrolene is a further anti-spastic drug in addition to baclofen. It inhibits calcium release from the sarcoplasmic reticulum by blocking ryanodine receptors and through this exerts an antispasmodic effect.

\section{Calcium-dependent Autoimmune}

\section{Mechanisms}

Autoreactive lymphocytes develop during the maturation process in the thymus when self-antigen recognizing thymocytes are not fully eliminated by the selection process. It has long been known that calcium-dependent processes also play an important role here [9]. The inhibition of activated T-cells by regulatory T-cells is also calcium-dependent. Recently, it was shown that regulatory T-cells of MS patients are less effective in blocking calcium signals in autoreactive, activated T-cells compared to regulatory T-cells of healthy control subjects [10]. Similar to neurons or cardio myocytes, lymphocytes and other electrically non-excitable cells express ion channels and transporters for the regulation of various physiological cell functions such as gene expression, apoptosis, proliferation, differentiation and migration. For example, during thymocyte selection or antigen-mediated activation of immune cells, a calcium influx is generated and maintained by a complex interaction of multiple channels [11]. As mentioned above, due to the importance of intracellular calcium as a central, secondary messenger, its level is also precisely regulated in T-lymphocytes. In recent years, fundamental advances have been made to identify the molecular components of the calcium signalling complex which is initiated upon T-cell activation. T-cell receptor (TCR) activation leads to the production of inositol 1,4,5-trisphosphate $\left(\mathrm{IP}_{3}\right)$ and calcium release from the ER. The subsequent activation of the calcium sensors stromal interaction molecules (STIM1) and STIM2 results in opening of calcium-activated calcium channels (calcium-release activated calcium (CRAC); $>$ Fig. 1). ORAl1 is the pore-forming subunit of these channels [12]. Free calcium in the cytosol induces various mechanisms urgently needed for the negative selection of autoreactive lymphocytes, immune cell activation upon antigen contact and much more.

This significance of these pathways has been known for a number of years. Patients with defective CRAC channels and inadequate calcium influx from the calcium stores (SOCE) suffer from a severe form of immune deficiency $[11,13]$. On the other hand, dysregulated calcium signals in T-cells can also lead to undesired autoreactive immune responses to self-antigens and trigger autoimmune inflammation. The group of CRAC channelopathies (diseases resulting from mutations in ORAI1 and STIM1 genes which interfere with or completely block CRAC channel function and SOCE) include severe combined immunodeficiency (SCID-like) diseases, autoimmunity, muscular hypotonia and ectodermal dysplasia. In animal models of MS as well as inflammatory intestinal diseases in which the genes for ORAI1, STIM1 or STIM2 have been genetically knocked out, it was found that the calcium sensors STIM1 and STIM2 play a central role in the function of proinflammatory immune cell subpopulations. In recent years, a large number of studies have been published which have investigated mutations in different genes involved in calcium regulation. This section focuses on the most recent publications on calcium homeostasis and autoimmunity.

Calcium plays an important role not only during the activation and maturation of lymphocytes against foreign antigens, but also in the differentiation of various immune cell subpopulations. For example, in a preclinical study, Kim and colleagues have shown that 


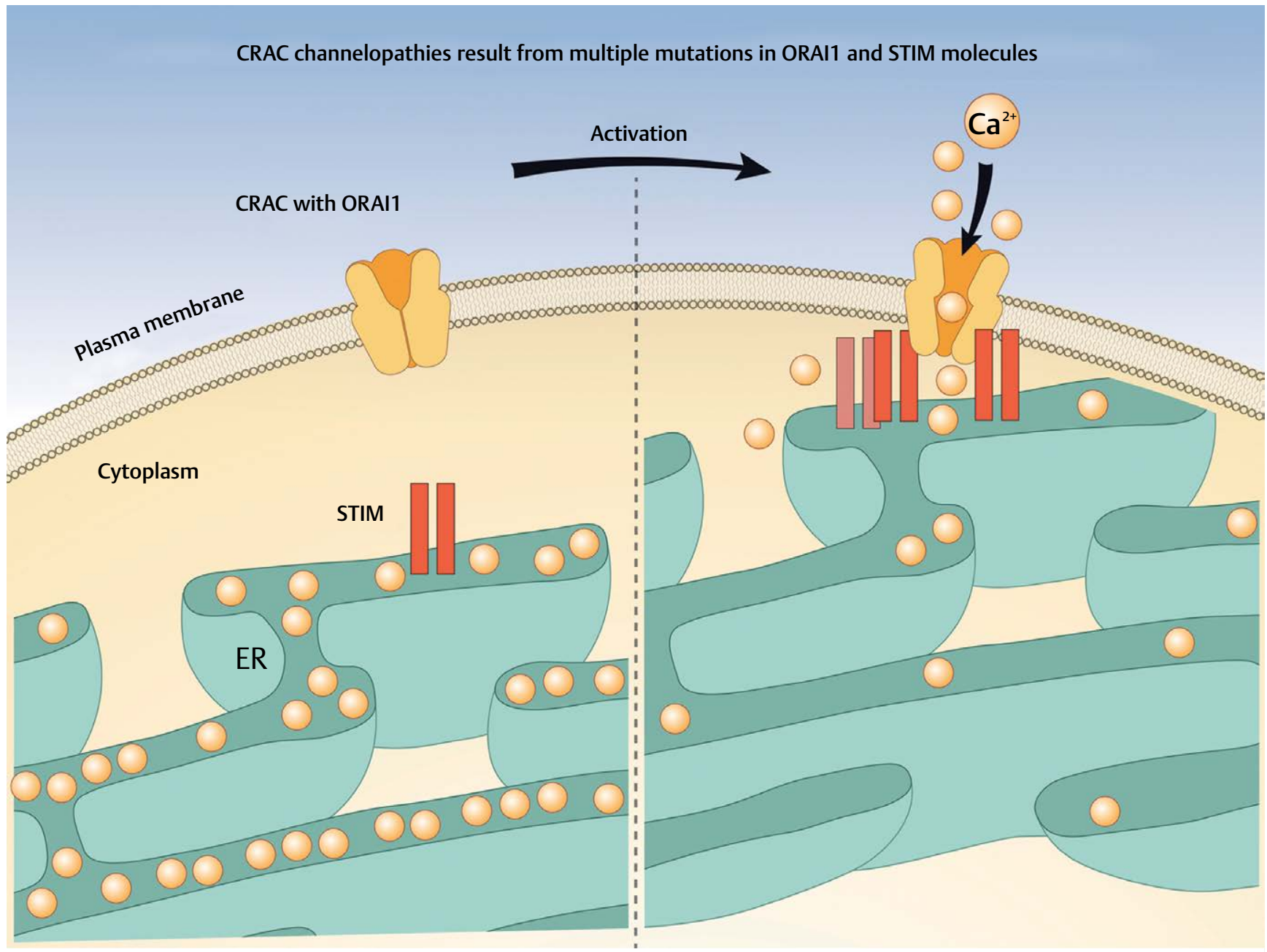

- Fig. 1 Store-operated Calcium Entry in T-lymphocytes. Upon release of calcium from the ER, STIM molecules aggregate in the ER membrane and activate CRAC channels in the cell membrane. This is followed by calcium influx from the extracellular space into the cell, thereby replenishing the intracellular calcium deposits. Mutations in STIM and ORAI1 genes lead to CRAC channelopathies. CRAC: Calcium release activated channels, ER: Endoplasmic reticulum, ORAI1: Calcium release-activated calcium channel protein 1, STIM1: Stromal interaction molecule 1.

ORAI1, the pore-forming subunit present in CRAC channels, is essential for the formation of T-cells that promote MS-like symptoms in the mouse model of MS. Blocking the ORAI1 pore, and thus the CRAC channels, significantly improved the clinical symptoms of the animals during in vivo experiments [16]. The group of CRAC channelopathies has been known and described for several years. However, there are still no CRAC-specific drugs for the treatment of immune disorders. Recently, several small molecules have been discovered and developed that block the function of CRAC channels and the intracellular calcium stores (SOCE) in micromolar concentrations [17]. For example, Chen and colleagues tested a chemical compound, RO2959, as a selective and potent CRAC channel blocker. They showed that the activation and effector functions of T cells were suppressed by application of the specific inhibitor [18]. Antibody-mediated approaches have also been investigated. The first monoclonal antibody ( $\mathrm{mAb}$ ) acting on ion channels in clinical development was directed against the pore-forming subunit of CRAC channels, ORAI1. The anti-ORAl1 mAb binds native ORAI 1 on lymphocytes and leads to the internalization of the channel. As a result, T-cell proliferation and cytokine production are inhibited in vitro. In vivo, anti-ORAI1 mAb is effective in a humanized T-cell-mediated graft-versus-host disease (GvHD) mouse model [19].

STIM1 acts as a sensor for intracellular calcium waste in the membrane of the ER. In addition to the ORAI1 gene, loss-of-function (LoF) mutations in the STIM1 gene are also a possible cause for the development of CRAC channelopathies. Fuchs and colleagues have investigated the function of the immune cells of two patients who had a homozygous R429C point mutation in the STIM1 gene. This mutation resulted in a blockade of the calcium influx from intracellular calcium stores. Investigations indicated that the patients suffered from a dampened immune regulation, which was (partly) due to an altered function of regulatory T-cells [20]. Regulatory T-cells, previously known as suppressor T-cells, are a subpopulation of T-cells that modulate the immune system, help to maintain tolerance to self-antigens, and prevent autoimmune diseases. It was assumed that also other immune cell subpopulations were affected by the defective STIM1-mediated calcium influx and thus contributed to disease pathology.

In addition to the LoF mutations in the STIM1 and ORAI1 genes, there are also various gain-of-function (GoF) mutations that cause 
a broad spectrum of disease entities, which surprisingly, are characterized by similar symptoms as the LoF mutations, among other things [21].

Recently, it has been shown that, besides through STIM and CRAC, calcium can also enter T-cells via a T-type calcium channel (low-voltage activated T-type, Cav3.1). Although Cav3.1 channels result in a substantial calcium flow under resting conditions, deletion of the channel in mice did not affect the TCR-initiated calcium influx. Nonetheless, Cav3.1-deficient mice did not develop any symptoms in the animal model for MS [22].

In addition, immune cells are also known to express members of the transient receptor potential melastatin (TRPM) channel family (TRPM2, TRPM7) [23, 24], which conduct various mono- and divalent cations (sodium, calcium, potassium). This family is activated by ligand binding and its members are mainly known for their role in perceiving cold (menthol) as well as taste. In a study by Wolf et al., it was shown that the calcium influx through TRPM2 channels does not contribute to an increase in the intracellular calcium level, which is absolutely necessary for receptor-mediated T-cell activation and triggering of effector functions [24]. The conditions under which calcium influx via TRPM2 channels plays a role is still not known. TRPM7 channels, another member of this family, are also expressed on T-lymphocytes. Their ion conductivity is characterized by a strong $\mathrm{pH}$ dependence and by the fact that their channel pore is blocked by a magnesium ion under physiological electrolyte conditions $[25,26]$. This suggests that the channel functions only under certain pathophysiological conditions (e. g. on immune cells in acidified, inflamed tissue).

In addition to the classical sources of calcium influx, new studies also link the inhibitory neurotransmitter GABA to the pathology of the MS animal model. However, in the literature, the effect of GABA is a matter of controversy. One study showed that GABA worsens the clinical symptoms in the MS model [27]. On the other hand, another recent study investigated the GABA-mediated mechanisms and showed that GABA application blocks the activation-induced calcium signals, resulting in an improvement in the clinical course of the disease $[28,29]$.

\section{Calcium-induced Loss of the Blood-Brain Barrier Integrity}

The blood-brain barrier (BBB) is the physical barrier between the periphery and the central nervous system. Its main task is to stabilize the micromilieu in the brain so that neural processes can proceed properly. This ensures the regulation of the nutrient transport into the CNS as well as the active blockage of infiltrating pathogens, toxins and autoreactive immune cells. The BBB is composed of endothelial cells surrounded by pericytes, smooth muscle cells and astroglial cells. This cell complex is also known as the neurovascular unit $[30,31]$.

On the molecular level, the barrier is formed both by adherens junctions (AJs) and tight junctions (TJs) ( $\triangleright$ Fig. 2). AJs connect adjacent endothelial cells and are formed by calcium-dependent transmembrane proteins called cadherins, which are connected intracellularly to the actin cytoskeleton by anchor proteins such as catenins and vinculins [32]. Apart from the AJ proteins, the T] pro- teins are essential for the barrier function of the BBB as they prevent the paracellular diffusion of molecules and ions. There are several TJ proteins that perform key functions to support the integrity of the BBB, such as occludin, claudin, junctional adhesion molecules (JAMs) and zona occludens 1-3 (ZOs). Claudins and occludin are composed of four transmembrane domains with intracellular $\mathrm{C}$ - and $\mathrm{N}$ - termini. Through extracellular loops they undergo hydrophilic interactions with the TJ proteins of neighbouring cells, thereby forming a physical barrier. To stabilize the T] complexes, occludin and claudin bind intracellularly to the cytoskeleton via the anchor proteins ZO 1-3. This junctional complex, which is specific for endothelial cells of the $B B B$, results in resistances of 1800-2000 $\Omega^{*} \mathrm{~cm}^{2}$ [33].

Indications for a loss of BBB integrity are found in many neurological diseases such as Alzheimer's, Parkinson's, epilepsy, cerebral ischemia and MS. In MS, BBB dysfunction and the accompanying transendothelial migration of activated, encephalitogenic lymphocytes are among the first observed cerebrovascular changes in the brain [34]. The signalling pathways underlying the impairment of the BBB have still been insufficiently investigated. However, it has long been known that changes in extra- and intracellular calcium concentrations play a central role in the loss of BBB integrity. Extracellular calcium decline leads to disruption in cell-cell and cell-extracellular matrix interactions. Intracellularly, an increase in calcium concentration initiates calcium-dependent signalling pathways, which ultimately induce restructuring of the cytoskeleton and concomitantly a reorganization of $\mathrm{T}$ J proteins resulting in a loss of BBB integrity [35].

Also at the BBB, several mechanisms are known which lead to an increase in intracellular calcium concentrations. Various growth factors, hormones, neurotransmitters and cytokines increase calcium levels after binding to their respective receptor on target cells. The activation of different, membrane-bound receptors, e. g. G-protein-coupled receptors (GPCRs) and tyrosine kinase receptors (TKs), mobilizes intracellular signalling molecules, which ultimately leads to the production of the secondary messenger $I P_{3} . I_{3}$ binds $I P_{3}$-receptors $\left(I P_{3} R\right)$ at the ER membrane, which induces the depletion of the calcium stores into the cytoplasm and subsequently causes an increase in intracellular calcium. In recent years, some vasoactive and inflammatory substances have been discovered, which result in increased cellular permeability and reduced transendothelial migration of autoreactive immune cells in neurological diseases. These include histamine, lysophosphatidic acid, thrombin, endothelins, bradykinin and the vascular endothelial growth factor (VEGF) [35]. New studies also suggest a protective role of the GPCRs cannabinoid receptor 2 (CB2R) in the function of the BBB under inflammatory conditions. Activation of CB2R increases resistance of endothelial cells and is accompanied by high regulation of TJ proteins. These results demonstrate that CR2B ligands could provide a new strategy for the treatment of neuroinflammatory diseases [36]. In an animal model of MS, it has also been shown that the integrity of the BBB is strictly regulated by inflammatory cytokines. The proinflammatory cytokines tumour necrosis factor $\alpha$ (TNF $\alpha$ ) and interleukin 6 (IL-6) induce BBB dysfunction by the dysregulation of $T$ J proteins [37]. In addition, inhibition of the calcium-dependent protein kinase $C \beta$ leads to a stabilization of the BBB in the disease model [38]. Recently, the expression of sphin- 


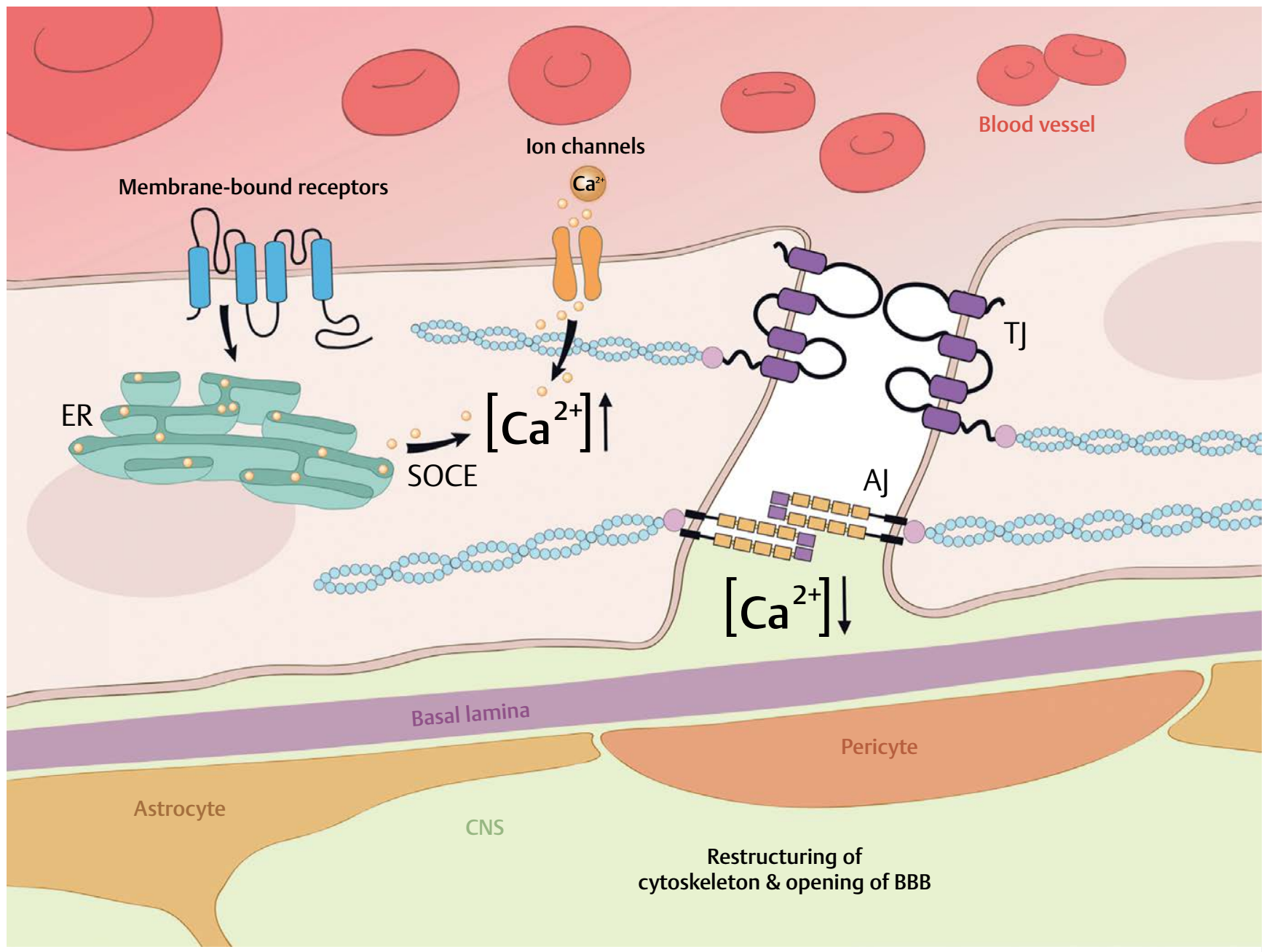

- Fig. 2 Calcium signal pathways in endothelial cells. Extracellular decrease in calcium concentration leads to the restructuring of AJ proteins and thus a loss in cell-cell contacts. An increase in intracellular calcium concentrations in endothelial cells is caused by 1 ) SOCE from the ER induced by the activation of membrane-bound receptors and 2) direct calcium influx via ion channels from the extracellular space. This results in a restructuring of the cytoskeleton and TJ proteins and opening of the BBB. AJ: Adherens junctions, BBB: Blood-brain barrier, ER: Endoplasmic reticulum, SOCE: Store-operated calcium entry, T]: Tight junctions

gosine-1-phosphate (S1P) receptor isoforms, which are targets of the MS drug fingolimod, has also been demonstrated on BBB endothelial cells and astrocytes. Activation of these isoforms results in an increase of the intracellular calcium level and thereby modulates BBB integrity. This could provide indication of an immune effect of fingolimod [39].

In addition to the calcium increase from intracellular deposits, direct calcium influx from the extracellular space via cation channels occurs in the endothelial cells of the BBB. Activation of N-methyl-D-aspartate (NMDA) receptors on microvascular endothelial cells has been shown to result in a decrease in transcellular electrical resistance caused by a change in occludin and ZO1 expression $[40,41]$, as well as the migration of monocytes across a human endothelial cell line [42]. In addition, the role of NMDA receptor-mediated calcium influx in a MS animal model has recently been clarified. The administration of idazoxan, a non-competitive inhibitor of the NMDA receptor, significantly reduces damage to the BBB and reverses the downregulation of T] proteins in an animal model [43]. A further study showed that the antibody-mediated blockade of the NMDA receptor significantly reduced the clinical symptoms in a MS animal model, and was accompanied by a reduced migration of lymphocytes into the CNS [44]. The transient receptor potential canonical (TRPC) channel family is another means by which calcium can enter the endothelial cells. The effects of the family members TRPC 1 and TRPC 4 on murine endothelial cells $[45,46]$ as well as of other TRPC-mediated mechanisms that impair the integrity of the BBB, are known for a while [46]. However, there has been no recent work in this field.

The extravasation of immune cells into the CNS across the BBB is also a calcium-dependent process. An important step in the migration of lymphocytes across the BBB is mediated via the interactions of LFA- 1 and MAC- 1 with the associated receptor ICAM-1, which is expressed on endothelial cells. Cross-linking of ICAM-1 molecules could induce the release of calcium from the ER in rat microvascular BBB endothelial cells [47]. In addition to ICAM-1, the adhesion molecule PECAM-1 also plays a role in the migration of immune cells across the BBB. PECAM- 1 is expressed both at the boundaries of two adjacent endothelial cells as well as on leuko- 


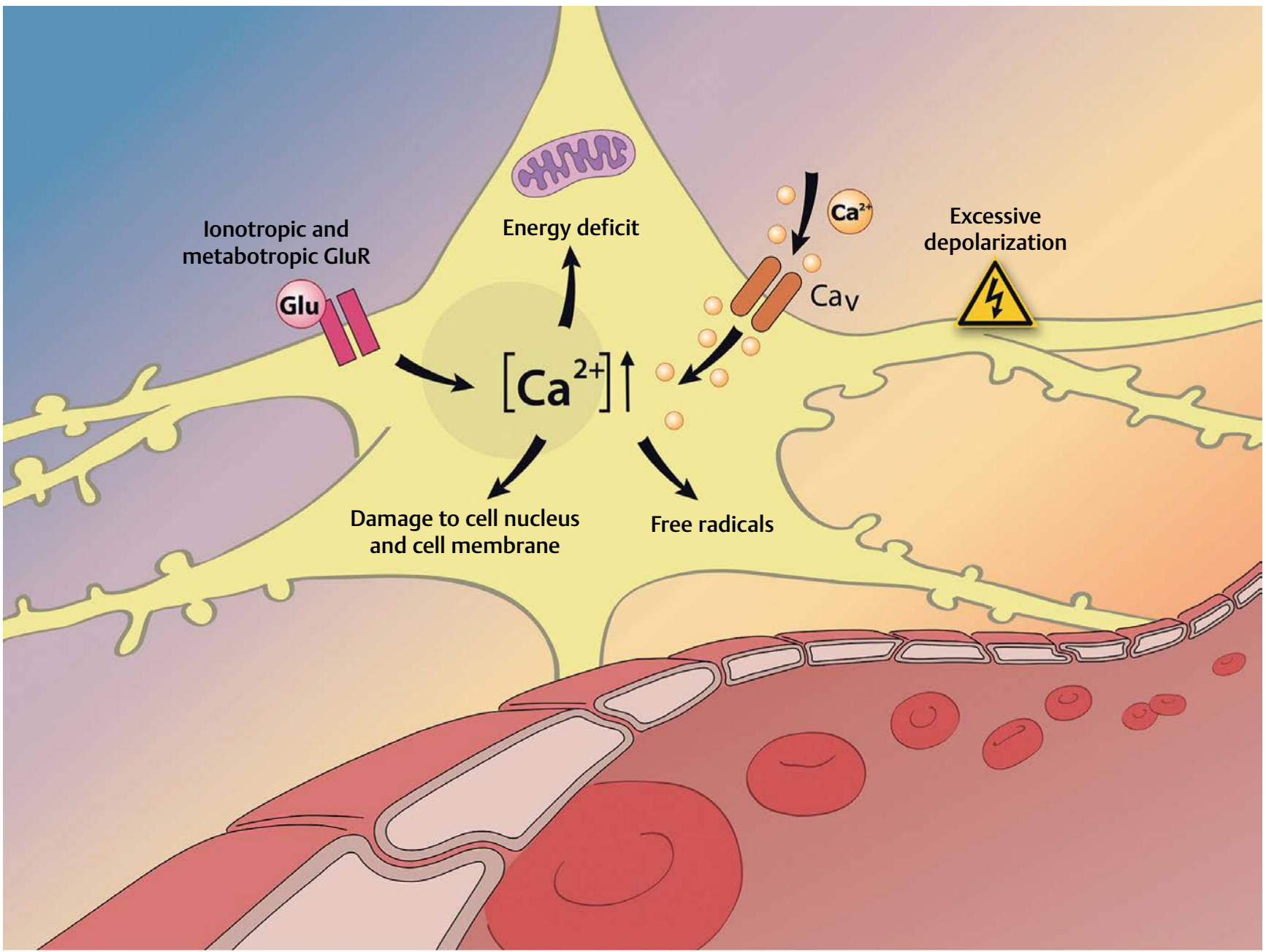

- Fig. 3 Calcium-induced neuronal cell death. An excess of extracellular glutamate leads to the opening of glutamate receptors, resulting in the influx of calcium into the cell and further depolarization of the neuron. Consequently, voltage-dependent calcium channels are opened and additional calcium can enter the cell. A permanently elevated intracellular calcium level damages the cell up to cell death.

cytes. Hydrophilic interactions between PECAM-1 on endothelial cells and leukocytes lead to an increase in the intracellular calcium level mediated by the ion channel TRPC6 [48]. It was also shown recently that the potassium channel TREK1 (Twik-related potassium channel 1) acts as a regulator of T-cell migration across the BBB in the MS mouse model [5].

All these mechanisms demonstrate that calcium plays an important role in BBB integrity, and the modulation of calcium-dependent processes offers potential for new therapeutic options for various neuroimmunological diseases.

\section{Calcium-dependent Neurodegenerative Processes in Central Nerve Cells}

There has been much discussion about how axons and neurons are damaged by autoimmune inflammation in the CNS. Meanwhile, experts agree that the loss of the myelin sheath alone cannot explain the extent of damage to axons in MS. There are numerous factors that contribute to neuronal and axonal damage. In MS and other neurodegenerative diseases, glutamate-induced excitotox- icity is a central downstream mechanism for neuronal injury leading to toxic increases in intraneuronal calcium [49]. Precisely regulated calcium movements across the plasma membrane and between intracellular compartments have a major influence on the basic functions of neurons, including regulation of neurite growth, synaptogenesis, synaptic transmission and plasticity, as well as cell survival. In classical excitotoxicity, increased extracellular glutamate induces a persistent depolarization of the neurons. This triggers a cascade of cellular events leading to cell death ( $>$ Fig. 3 ). Depolarization is initiated primarily by the activation of AMPA receptors and subsequently by activation of voltage-dependent sodium channels, resulting in sodium influx and further depolarization of the cell membrane. If the cell is chronically depolarized, the NMDA receptor loses its magnesium block and can be activated via binding of synaptic glutamate. NMDA receptor activation is an important source of calcium entry into the cell. Intracellular calcium levels are very low (nM range) under physiological resting conditions. If the cell is excessively depolarized, the calcium level will rise continuously. After NMDA receptor activation, the most important sources of increased intracellular free calcium are entry via voltage-dependent calcium channels after initial depolarization. This 
calcium influx, however, is only temporary, since both voltage-dependent calcium channels and NMDA receptors deactivate despite continuous membrane depolarization, halting calcium influx. In addition to the ionotropic AMPA and NMDA receptors, glutamate can also bind to metabotropic receptors, which upon activation, can trigger the release of calcium via the incorporation of various enzymes and signalling molecules (phospholipase $C, I_{3}$ ) from the ER [49] and thus contribute to the fatal, continuous increase of the calcium level in the cell. In addition to glutamate receptors and voltage-controlled calcium channels, neurons, like the immune and endothelial cells, additionally express non-selective, predominantly ligand-activated cation channels, through which calcium can enter the cells. However, relative to the two main pathways via glutamate receptor channels and calcium channels, these channels provide only a small fraction of the neurodegenerative processes relevant for $\mathrm{MS}$.

Induction of caspase-dependent apoptosis is one of the harmful events caused by excess calcium in the cytosol [50]. Another detrimental result of excess calcium in the cytosol is the opening of the mitochondrial permeability transition pore, a pore in the membranes of the mitochondria that opens when the organelles absorb too much calcium. Opening the pore can cause mitochondria to swell and release reactive oxygen species as well as other proteins that can initiate apoptosis. The pore can also cause mitochondria to release more calcium. In addition, the production of adenosine triphosphate (ATP) can be stopped and ATP synthase can start to hydrolyze ATP, thus reducing it [51]. It is clear that the classical theory of excitotoxicity via glutamate is much more complex, and presumably there are significantly more harmful mechanisms than previously known [49]. Also, with the knowledge that to date no effective therapy strategy could be developed to significantly reduce neuronal excitotoxicity, numerous research groups continue to study neuronal calcium homeostasis under physiological and pathophysiological conditions.

Thus, in a recent study using modern imaging technology, inflammation-induced changes of the calcium level in the axons of neurons were investigated in mice [52]. The authors demonstrated an increase in free calcium in axons and neurons of autoimmune-inflammatory lesions compared to those in non-inflamed areas. These relative calcium increases were associated with immune-neuronal interactions.

A recently published article describes a phenomenon that combines various neurodegenerative diseases: the misfolding and aggregation of the key proteins $\alpha$-synuclein and $\beta$-amyloid [53]. Although the primary targets for the two proteins are different, they both share a common mechanism that involves formation of porelike structures in the plasma membrane, resulting in the free exchange of proteins, ions and substances. Among other things, this leads to a disturbance of the calcium content, the mitochondria and to damage by free radicals. The causes of the formation of these oxygen radicals should also be investigated in a further study. Using intravital microscopy, the authors observed an elevated calcium level in the neurons in the cerebral arteries with oxidative stress, which was interpreted as subclinical neuronal dysfunction and the onset of neuronal damage [54].

It has been known for a few years that the proinflammatory platelet activation factor (PAF) is increased a thousand-fold in the
CSF of MS patients [55], resulting in the modulation of signals, including the production of nitric oxides, mitochondrial calcium influx and caspase activation. Surprisingly, recent studies demonstrated a concrete link between proteins of the coagulation system and the pathophysiology of MS [56-58]. Likewise, a recent study by Bellizzi et al. showed that the PAF receptor in an animal model of MS mediates the over-excitation of hippocampal neurons and thus contributes to neuronal damage [59]. This is particularly interesting because the understanding of the mechanisms of the degeneration of the grey matter is still limited. However, this is indispensable for future development of treatments for progressive disability and cognitive impairment in MS. Thus, studies are directed toward the development of substances that are directly neuroprotective. Fingolimod and dimethyl fumarate were injected in order to subsequently measure the intraneuronal calcium level in a living animal by means of microscopy. Direct CNS administration of these MS therapeutics has significantly delayed or reduced glutamate-induced overexcitation, but could not prevent neuronal calcium increase or morphological changes [60].

Optic nerve neuritis, a typical manifestation in the disease pattern of multiple sclerosis, is associated with the axonal degeneration of the optic nerve (ON) and apoptosis of retinal ganglion cells (RGC). One study demonstrated that inhibition of voltage-dependent N-type calcium channels in an animal model of MS leads to a significant reduction of axonal degeneration [61]. Furthermore, the calcium-induced damage to optic nerve and retinal ganglion cells was shown to be mediated by calpain, a calcium-dependent, non-lysosomal cysteine protease, and that blockade prevented RGCs against degeneration [62]. Recently the role of NMDA receptors on retinal ganglion cells was made clear. Inhibition of these receptors in an animal model of MS results in protection of retinal ganglion cells and axons and reduced demyelination of the optic nerve [63].

\section{Summary}

In conclusion, understanding of calcium-dependent processes in the context of autoimmune diseases and in particular, MS, is continuously improving. It has been shown that regulation of calcium via various mechanisms is involved in all relevant steps of MS pathogenesis, such as immunosensitivity activation and migration, the regulation of BBB integrity and neurodegeneration itself. Specific pharmaceuticals (small molecules, therapeutic antibodies) are being tested in preclinical models and should be transferred to the clinic using translational approaches. However, the use of currently available calcium channel blockers for specific MS therapy cannot be recommended. Therefore, currently authorized drugs should be used strictly according to their approved indication.

\section{Conflict of Interest}

P. Hundehege has received fees for lectures and travel expenses for attending meetings and has received financial support from Merck Serono and Novartis. L. Epping declares no conflicts of interest. S. G. Meuth has received fees for lectures and travel expenses for attending meetings and has received financial support from Almirall, 
Bayer Health Care, Biogen, Diamed, Fresenius Medical Care, Genzyme, Merck Serono, Novartis, Novo Nordisk, ONO Pharma, Roche, Sanofi-Aventis and Teva.

\section{References}

[1] Frohman EM, Racke MK, Raine CS. Multiple sclerosis-the plaque and its pathogenesis. N Engl J Med 2006; 354: 942-955

[2] Mahad DH, Trapp BD, Lassmann H. Pathological mechanisms in progressive multiple sclerosis. Lancet Neurol 2015; 14: 183-193

[3] Hemmer B, Kerschensteiner M, Korn T. Role of the innate and adaptive immune responses in the course of multiple sclerosis. Lancet Neurol 2015; 14: 406-419

[4] Mallucci G, Peruzzotti-jametti L, Bernstock JD et al. The role of immune cells, glia and neurons in white and gray matter pathology in multiple sclerosis. Prog Neurobiol 2015; 127-128: 1-22

[5] Bittner S, Ruck T, Schuhmann M et al. Endothelial TWIK-related potassium channel-1 (TREK1) regulates immune-cell trafficking into the CNS. Nat Med 2013; 19: 1161-1165

[6] Breuer J, Schwab N, Schneider-Hohendorf T et al. Ultraviolet B light attenuates the systemic immune response in central nervous system autoimmunity. Ann Neurol 2014; 75: 739-758

[7] Clapham DE. Calcium signaling. Cell 2007; 131: 1047-1058

[8] Wu ZZ, Li DP, Chen SR et al. Aminopyridines potentiate synaptic and neuromuscular transmission by targeting the voltage-activated calcium channel beta subunit. J Biol Chem 2009; 284: 36453-36461

[9] Sebzda E, Mariathasan S, Ohteki T et al. Selection of the T cell repertoire. Annu Rev Immunol 1999; 17: 829-874

[10] Schwarz A, Schumacher M, Pfaff D et al. Fine-tuning of regulatory T cell function: the role of calcium signals and naive regulatory $T$ cells for regulatory T cell deficiency in multiple sclerosis. J Immunol 2013; 190: 4965-4970

[11] Feske S, Wulff H, Skolnik EY. Ion channels in innate and adaptive immunity. Annu Rev Immunol 2015; 33: 291-353

[12] Hogan PG, Lewis RS, Rao A. Molecular basis of calcium signaling in lymphocytes: STIM and ORAI. Annu Rev Immunol 2010; 28: 491-533

[13] Feske S. Immunodeficiency due to defects in store-operated calcium entry. Ann NY Acad Sci 2011; 1238: 74-90

[14] Schuhmann M, Stegner D, Berna-Erro A et al. Stromal interaction molecules 1 and 2 are key regulators of autoreactive $T$ cell activation in murine autoimmune central nervous system inflammation. J Immunology 2010; 184: 1536-1542

[15] Ma J, McCarl C-A, Khalil S et al. T-cell-specific deletion of STIM1 and STIM2 protects mice from EAE by impairing the effector functions of Th1 and Th17 cells. Eur I Immunology 2010; 40: 3028-3042

[16] Kim KD, Srikanth S, Tan YV et al. Calcium signaling via Orai1 is essential for induction of the nuclear orphan receptor pathway to drive Th17 differentiation. J Immunol 2014; 192: 110-122

[17] Tian C, Du L, Zhou Y et al. Store-operated CRAC channel inhibitors: opportunities and challenges. Future Med Chem 2016; 8: 817-832

[18] Chen G, Panicker S, Lau KY et al. Characterization of a novel CRAC inhibitor that potently blocks human $T$ cell activation and effector functions. Mol Immunol 2013; 54: 355-367

[19] Cox JH, Hussell S, Sondergaard $\mathrm{H}$ et al. Antibody-mediated targeting of the Orai1 calcium channel inhibits T cell function. PLoS One 2013; 8: e82944

[20] Fuchs S, Rensing-Ehl A, Speckmann C et al. Antiviral and regulatory $T$ cell immunity in a patient with stromal interaction molecule 1 deficiency. J Immunol 2012; 188: 1523-1533
[21] Lacruz RS, Feske S. Diseases caused by mutations in ORAI1 and STIM1. Ann N Y Acad Sci 2015; 1356: 45-79

[22] Wang $H$, Zhang X, Xue L et al. Low-voltage-activated CaV3.1 calcium channels shape thelper cell cytokine profiles. Immunity 2016; 44 782-794

[23] Ehling P, Meuth P, Eichinger $P$ et al. Human T cells in silico: Modelling their electrophysiological behaviour in health and disease. J Theor Biol 2016; 404: 236-250

[24] Wolf IM, Diercks BP, Gattkowski E et al. Frontrunners of T cell activation: Initial, localized $\mathrm{Ca} 2+$ signals mediated by NAADP and the type 1 ryanodine receptor. Sci Signal 2015; 8: ra102

[25] Chokshi R, Matsushita M, Kozak JA. Sensitivity of TRPM7 channels to $\mathrm{Mg} 2+$ characterized in cell-free patches of Jurkat T lymphocytes. Am J Physiol Cell Physiol 2012; 302: C1642-1651

[26] Chokshi R, Matsushita M, Kozak J. Detailed examination of Mg2 + and $\mathrm{pH}$ sensitivity of human TRPM7 channels. Am J Physiol Cell Physiol 2012; 302: 11

[27] Carmans S, Hendriks J], Slaets $\mathrm{H}$ et al. Systemic treatment with the inhibitory neurotransmitter gamma-aminobutyric acid aggravates experimental autoimmune encephalomyelitis by affecting proinflammatory immune responses. J Neuroimmunol 2013; 255: 45-53

[28] Prud'homme G], Glinka Y, Wang Q. Immunological GABAergic interactions and therapeutic applications in autoimmune diseases. Autoimmun Rev 2015; 14: 1048-1056

[29] Alam S, Laughton DL, Walding A et al. Human peripheral blood mononuclear cells express GABAA receptor subunits. Mol Immunol 2006; 43: 1432-1442

[30] Abbott N], Patabendige AA, Dolman DE et al. Structure and function of the blood-brain barrier. Neurobiol Dis 2010; 37: 13-25

[31] Hawkins BT, Davis TP. The blood-brain barrier/neurovascular unit in health and disease. Pharmacol Rev 2005; 57: 173-185

[32] Hartsock A, Nelson W]. Adherens and tight junctions: Structure, function and connections to the actin cytoskeleton. Biochim Biophys Acta 2008; 1778: 660-669

[33] Coisne C, Engelhardt B. Tight junctions in brain barriers during centra nervous system inflammation. Antioxid Redox Signal 2011; 15: 1285-1303

[34] Minagar A, Alexander JS. Blood-brain barrier disruption in multiple sclerosis. Mult Scler 2003; 9: 540-549

[35] De Bock M, Wang N, Decrock E et al. Endothelial calcium dynamics, connexin channels and blood-brain barrier function. Prog Neurobiol 2013; 108: 1-20

[36] Ramirez SH, Hasko J, Skuba A et al. Activation of cannabinoid receptor 2 attenuates leukocyte-endothelial cell interactions and blood-brain barrier dysfunction under inflammatory conditions. J Neurosci 2012; 32: 4004-4016

[37] Rochfort KD, Cummins PM. The blood-brain barrier endothelium: a target for pro-inflammatory cytokines. Biochem Soc Trans 2015; 43: 702-706

[38] Lanz TV, Becker S, Osswald M et al. Protein kinase Cbeta as a therapeutic target stabilizing blood-brain barrier disruption in experimental autoimmune encephalomyelitis. Proc Natl Acad Sci U S A 2013; 110: 14735-14740

[39] Prager B, Spampinato SF, Ransohoff RM. Sphingosine 1-phosphate signaling at the blood-brain barrier. Trends Mol Med 2015; 21: 354-363

[40] Chen JT, Chen TG, Chang YC et al. Roles of NMDARs in maintenance of the mouse cerebrovascular endothelial cell-constructed tight junction barrier. Toxicol 2016; 339: 40-50

[41] Neuhaus W, Freidl M, Szkokan P et al. Effects of NMDA receptor modulators on a blood-brain barrier in vitro model. Brain Res 2011; 1394: 49-61 
[42] Reijerkerk A, Kooij G, van der Pol SM et al. The NR1 subunit of NMDA receptor regulates monocyte transmigration through the brain endothelial cell barrier. J Neurochem 2010; 113: 447-453

[43] Wang XS, Fang HL, Chen Y et al. Idazoxan reduces blood-brain barrier damage during experimental autoimmune encephalomyelitis in mouse. Eur J Pharmacol 2014; 736: 70-76

[44] Macrez R, Ortega MC, Bardou I et al. Neuroendothelial NMDA receptors as therapeutic targets in experimental autoimmune encephalomyelitis. Brain 2016; 139: 2406-2419

[45] Balbuena P, Li W, Rzigalinski BA et al. Malathion/oxon and lead acetate increase gene expression and protein levels of transient receptor potential canonical channel subunits TRPC1 and TRPC 4 in rat endothelial cells of the blood-brain barrier. Int J Toxicol 2012; 31: 238-249

[46] Hicks K, O'Neil RG, Dubinsky WS et al. TRPC-mediated actin-myosin contraction is critical for BBB disruption following hypoxic stress. Am J Physiol Cell Physiol 2010; 298: C1583-1593

[47] Etienne-Manneville S, Manneville JB, Adamson P et al. ICAM-1-coupled cytoskeletal rearrangements and transendothelial lymphocyte migration involve intracellular calcium signaling in brain endothelial cell lines. J Immunol 2000; 165: 3375-3383

[48] Weber EW, Han F, Tauseef M et al. TRPC6 is the endothelial calcium channel that regulates leukocyte transendothelial migration during the inflammatory response. J Exp Med 2015; 212: 1883-1899

[49] Kostic M, Zivkovic N, Stojanovic I. Multiple sclerosis and glutamate excitotoxicity. Rev Nneurosci 2013; 24: 71-88

[50] Dutta R, Trapp BD. Mechanisms of neuronal dysfunction and degeneration in multiple sclerosis. Prog Neurobiol 2011; 93: 1-12

[51] Stavrovskaya IG, Kristal BS. The powerhouse takes control of the cell: Is the mitochondrial permeability transition a viable therapeutic target against neuronal dysfunction and death? Free Radic Biol Med 2005; 38: 687-697

[52] Siffrin V, Birkenstock J, Luchtman DW et al. FRET based ratiometric $\mathrm{Ca}(2+)$ imaging to investigate immune-mediated neuronal and axonal damage processes in experimental autoimmune encephalomyelitis. | Neurosci Methods 2015; 249: 8-15
[53] Angelova PR, Abramov AY. Alpha-synuclein and beta-amyloid - different targets, same players: calcium, free radicals and mitochondria in the mechanism of neurodegeneration. Biochem Biophys Res Commun 2016; 483: 1110-1115

[54] Radbruch H, Bremer D, Guenther R et al. Ongoing oxidative stress causes subclinical neuronal dysfunction in the recovery phase of EAE. Front Immunol 2016; 7: 92

[55] Callea L, Arese M, Orlandini A et al. Platelet activating factor is elevated in cerebral spinal fluid and plasma of patients with relapsing-remitting multiple sclerosis. J Neuroimmunol 1999; 94: 212-221

[56] Göbel K, Pankratz S, Asaridou CM et al. Blood coagulation factor XII drives adaptive immunity during neuroinflammation via CD87-mediated modulation of dendritic cells. Nat Commun 2016; 7: 11626

[57] Göbel K, Kraft P, Pankratz $S$ et al. Prothrombin and factor $X$ are elevated in multiple sclerosis patients. Ann Neurol 2016; 80: 946-951

[58] Pankratz S, Bittner S, Kehrel BE et al. The inflammatory role of platelets: translational insights from experimental studies of autoimmune disorders. Int J Mol Sci 2016 doi: 10.3390/ijms17101723

[59] Bellizzi M], Geathers JS, Allan KC et al. Platelet-activating factor receptors mediate excitatory postsynaptic hippocampal injury in experimental autoimmune encephalomyelitis. J Neurosci 2016; 36: 1336-1346

[60] Luchtman D, Gollan R, Ellwardt E et al. In vivo and in vitro effects of multiple sclerosis immunomodulatory therapeutics on glutamatergic excitotoxicity. J Neurochem 2016; 136: 971-980

[61] Gadjanski I, Boretius S, Williams SK et al. Role of n-type voltage-dependent calcium channels in autoimmune optic neuritis. Ann Neurol 2009; 66: 81-93

[62] Hoffmann DB, Williams SK, Bojcevski J et al. Calcium influx and calpain activation mediate preclinical retinal neurodegeneration in autoimmune optic neuritis. J Neuropathol Exp Neurol 2013; 72: 745-757

[63] Sühs KW, Fairless R, Williams SK et al. N-methyl-D-aspartate receptor blockade is neuroprotective in experimental autoimmune optic neuritis. J Neuropathol Exp Neurol 2014; 73: 507-518 